\title{
Atomic-scale structure of the fivefold surface of an AlPdMn quasicrystal: A quantitative x-ray photoelectron diffraction analysis
}

\author{
Jin-Cheng Zheng, ${ }^{1}$ C. H. A. Huan, ${ }^{1}$ A. T. S. Wee, ${ }^{1}$ M. A. Van Hove, ${ }^{2,3,4}$ C. S. Fadley, ${ }^{2,4}$ F. J. Shi, ${ }^{2}$ E. Rotenberg, ${ }^{3}$ \\ S. R. Barman, ${ }^{5}$ J. J. Paggel ${ }^{6}{ }^{K}$. Horn, ${ }^{7}$ Ph. Ebert ${ }^{8}$ and K. Urban ${ }^{8}$ \\ ${ }^{1}$ Department of Physics, National University of Singapore, Lower Kent Ridge Road, Singapore 119260 \\ ${ }^{2}$ Materials Sciences Division, Lawrence Berkeley National Laboratory, Berkeley, California 94720, USA \\ ${ }^{3}$ Advanced Light Source, Lawrence Berkeley National Laboratory, Berkeley, California 94720, USA \\ ${ }^{4}$ Department of Physics, University of California at Davis, Davis, California 95616, USA \\ ${ }^{5}$ Inter-University Consortium for DAEF, Indore, India \\ ${ }^{6}$ Fachbereich Physik der Freien Universität, Berlin, Germany \\ ${ }^{7}$ Fritz-Haber-Institut der Max-Planck-Gesellschaft, Berlin, Germany \\ ${ }^{8}$ Institut für Festkörperforschung des Forschungszentrums, Jülich, Germany
}

(Received 17 March 2003; revised manuscript received 17 February 2004; published 16 April 2004)

\begin{abstract}
The atomic-scale structure of the fivefold symmetric surface of an AlPdMn quasicrystal is investigated quantitatively by comparing $\mathrm{x}$-ray photoelectron diffraction simulations to experiment. The observed fivefold symmetry of the diffraction patterns indicates that the surface is quasicrystalline with no hint of a reconstruction from the bulk structure. In analyzing the experimental data, many possible bulk terminations have been tested. Those few that fit best to the data have in common that they contain an Al-rich surface layer followed by a dense mixed $\mathrm{Al} / \mathrm{Pd} / \mathrm{Mn}$ layer. These best terminations, while not identical to each other, are suggested to form terraces coexisting on a real surface. Structural relaxations of the quasicrystal surface are also analyzed: mixing several best-fit terminations gives average best-fit interlayer spacing changes of $\Delta d_{12}=-0.057 \AA$ and $\Delta d_{24}=+0.159 \AA$. These results are in good agreement with a prior structure determination by low-energy electron diffraction on a sample that was prepared in a different manner.
\end{abstract}

DOI: 10.1103/PhysRevB.69.134107

PACS number(s): 68.35.Bs, 68.47.De, 68.49.Jk

\section{INTRODUCTION}

Although much is understood about the bulk atomic-scale structure of quasicrystals, ${ }^{1-3}$ the determination of their surface structures presents important challenges. ${ }^{4}$ Information about the surface structure, composition, chemistry, topology, and possible surface reconstructions is a prerequisite to understanding the recent findings that quasicrystalline surfaces and coatings exhibit high hardness, low surface friction, and high oxidation resistance, i.e., properties important for many technological applications. Moreover, the complex processes which favor quasicrystalline ordering are probably also related to the interaction at the growth front, i.e., the surface.

The surface structures of both icosahedral and decagonal alloys have been examined previously by scanning tunneling microscopy (STM) and low-energy electron diffraction (LEED), among many techniques. ${ }^{5-14}$ Earlier LEED studies were limited to observations and discussions of the symmetry and spacing of the diffraction spots, addressing the question of whether the surface retains the quasicrystallinity of the bulk. More recently, Gierer et al. ${ }^{4}$ used dynamical LEED to obtain atomic-scale information on the surface structure and composition of the fivefold surface of AlPdMn: that work was performed with a sample differently prepared from that in the present study, but the expectation is that the structures should nevertheless be similar. The LEED study favored a mix of several relaxed, bulklike terminations, with a dense Al-rich layer on top followed by a layer with a composition of about $50 \% \mathrm{Al}$ and $50 \% \mathrm{Pd}$. The spacing between these two topmost layers ( $0.48 \AA$ in the bulk) was found to be contracted by about $0.1 \AA$ from the bulk value, and the two-dimensional density of these two almost coplanar layers taken together was similar to that of one close-packed atomic layer of an $\mathrm{Al}(111)$ surface. The more recent, and more qualitative, studies are largely consistent with the LEED results ${ }^{4}$ and with the conclusions of the present detailed study.

In this work, the surface structure of an AlPdMn quasicrystal has been studied by means of core-level x-ray photoelectron diffraction (XPD). ${ }^{15-17}$ XPD is similar to LEED in that the photoemitted electrons may undergo elastic scattering from the atoms of the crystal, and the interference between the direct and the scattered waves gives rise to diffraction patterns that contain structural information. However, since each element has a unique photoelectron spectrum, it is almost always possible to find a core peak at a kinetic energy specific to each element of the system under consideration. It is then also possible in many cases to localize the origin of the signal in a subset of sites of the crystal, so that different features of the system may be emphasized. Compared to LEED, XPD is more sensitive to lateral displacements of atoms parallel to the surface, since the electron momentum transfer in XPD under the usual conditions is less parallel to the surface. The complementary differences between XPD and LEED allow us to derive a more complete picture of the system and obtain a better understanding of the surface structure of the AlPdMn quasicrystal.

The organization of this paper is as follows. In Sec. II the experimental measurements of XPD from $\mathrm{Al} 2 p$ and $\mathrm{Pd} 3 d$ in an AlPdMn quasicrystal are presented. Section III describes the computational techniques used in theoretical 


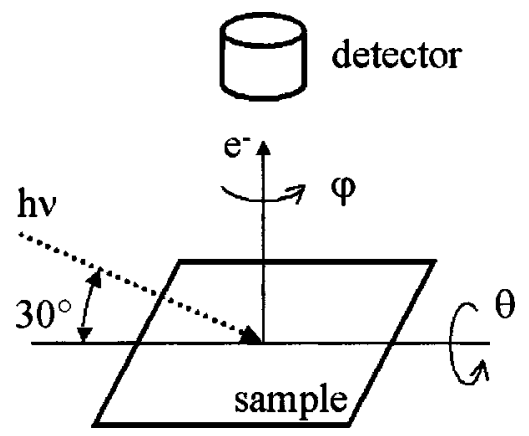

FIG. 1. Experimental photoemission geometry.

simulations of XPD, based on multiple scattering theory. In Sec. IV, the modeling of a quasicrystal surface for XPD simulations is discussed in detail. Finally, the quantitative analysis and a discussion of the quasicrystal surface structure are presented in Sec. V, while our conclusions are given in Sec. VI.

\section{EXPERIMENTAL PROCEDURES}

The measurements were performed with a standard laboratory x-ray $\mathrm{Mg} K \alpha$ source $(h \nu=1253.6 \mathrm{eV})$ and a Physical Electronics Omni IV spectrometer system. The experimental geometry is shown in Fig. 1. The photoelectrons were energy analyzed by an electrostatic hemispherical electron energy analyzer (PHI model 10-360) and detected by a channelplate array. The angular resolution was about $\pm 0.7^{\circ}$. XPD was performed by rotating the sample, and the raw signal was obtained by integrating a fixed energy window centered on each core level and subtracting a similar window taken from the nearby background.

Data were recorded from a prepolished disk of AlPdMn in the fivefold orientation, from a boule grown by the Czochralski method in the Forschungszentrum Jülich. The sample, with a diameter of $10 \mathrm{~mm}$ and a thickness of $2 \mathrm{~mm}$, had a bulk composition of $70.5 \%, \mathrm{Al}, 21 \% \mathrm{Pd}$, and $8.5 \% \mathrm{Mn}$. The surface was cleaned by neon ion sputtering and prolonged annealing to about $800 \mathrm{~K}$. Cleanliness of the sample was evaluated from the absence of an oxide shoulder on the Al $2 p$ peak, recorded under conditions of extreme surface sensitivity, and the absence of other contaminants as identified from the entire photoelectron spectrum.

Sample cleaning was repeated about every $2-3 \mathrm{~h}$. Since it is well known that ion bombardment can lead to surface depletion of specific alloy components, the intensity of $\mathrm{Al}$, $\mathrm{Pd}$, and Mn core-level lines was recorded after the preparation process. The relative intensity of these lines was compared with that recorded from a sample of the same boule, where the surface was prepared by mechanical cleaving in ultrahigh vacuum. These data served as a reference for the bulk composition. It was found that the annealing process recovered a composition in the surface region probed by the photoemission experiment which was very close to that of the bulk.

This sample exhibited a fivefold LEED pattern ${ }^{4}$ and a fivefold photoelectron diffraction pattern from the $\mathrm{Al} 2 p$, Pd
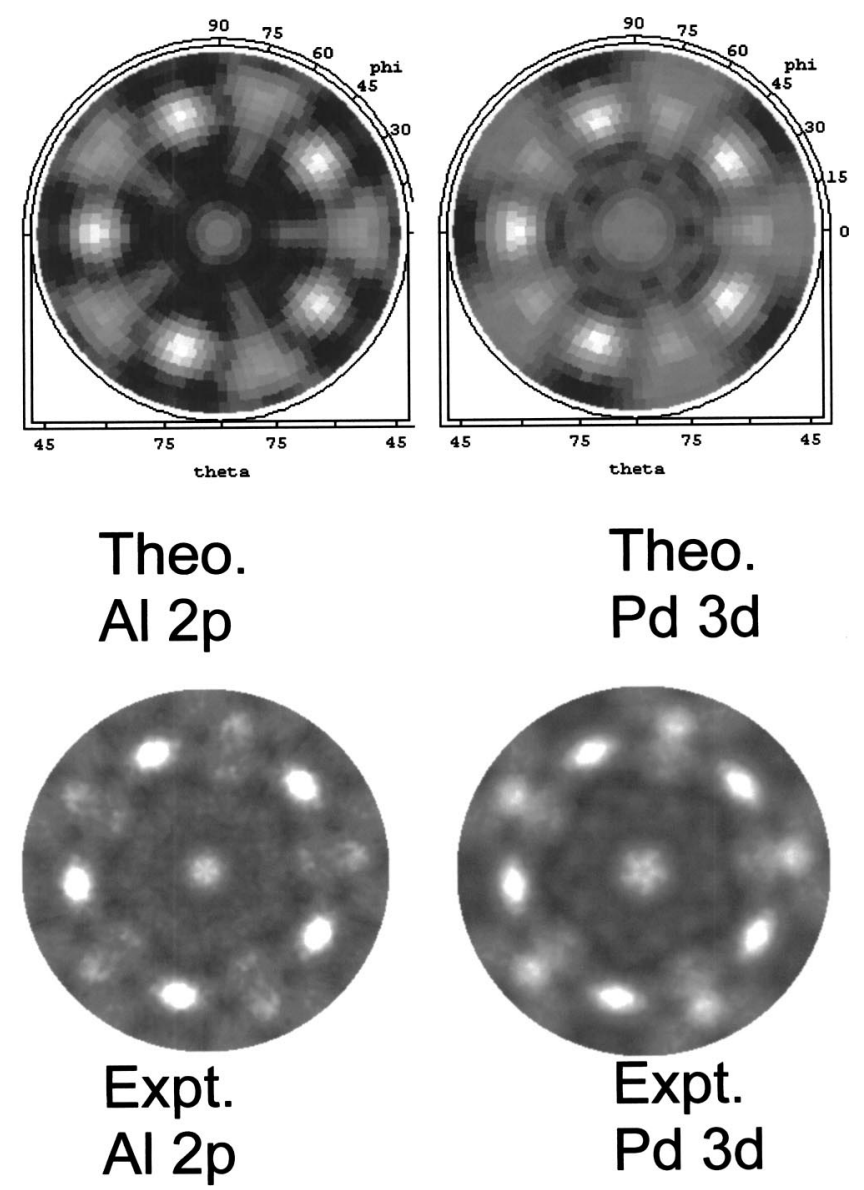

FIG. 2. Comparison between optimized simulated (top) and experimental (bottom) XPD for $\mathrm{Al} 2 p$ (left) and Pd $3 d$ (right). In the theoretical patterns of this and other figures, the polar angle labeling refers to the surface plane, so that the data range from the surface normal at $90^{\circ}$ to $45^{\circ}$ from the surface plane.

$3 d$, and Mn $2 p$ lines, excited with $\operatorname{Mg} K \alpha$ radiation, as reported in the literature. ${ }^{18}$

The results of angle-scanned XPD (polar angle up to $\theta_{\max }=45^{\circ}$ from the surface normal) from $\mathrm{Al} 2 p$ and $\mathrm{Pd} 3 d$ emission, together with optimized simulations, are shown in Fig. 2. The photoelectron energy was $1181 \mathrm{eV}$ for $\mathrm{Al} 2 p$ and $919 \mathrm{eV}$ for $\mathrm{Pd} 3 d$. The raw data were acquired for $220^{\circ}$ azimuthal range and were fully consistent with the fivefold symmetry of the AlPdMn quasicrystal. To reduce noise, the data shown in Fig. 2 were therefore rotationally averaged according to this symmetry. The optimization procedure for the theoretical simulations is discussed in detail in Sec. V below.

\section{METHOD OF CALCULATION}

The multiple scattering calculation of diffraction (MSCD) package developed by Chen et al. ${ }^{15}$ is used for the analysis of the XPD data. This program simulates the elemental and state-specific core-level photoelectron diffraction pattern from an atomic cluster that represents a surface. It is based on multiple scattering theory with the Rehr-Albers (RA) separable representation of spherical-wave propagators, and 
is used to produce structures yielding best fits to the experimental data. To model the use of unpolarized radiation, the results of MSCD for two orthogonal linear polarizations were averaged.

The inelastic mean free path of about $12 \AA$ was estimated with a formula (proportional to $E^{1 / 2}$ ) derived by Seah and Dench using experimental attenuation lengths for several solid elements. ${ }^{19}$

Cluster sizes of about 100-150 atoms have been shown to be adequate for surfaces of metals and other materials, such as $\mathrm{W}(110), \mathrm{O} / \mathrm{W}(110), \mathrm{Li} / \mathrm{Al}(111)$, and $\mathrm{MnO}(100) .{ }^{15}$ We have used somewhat larger cluster sizes of about 200-300 atoms for the modeling of the quasicrystal surface: our tests (described below) show that these are sufficient.

A major challenge in studying quasicrystal surfaces by quantitative XPD lies in how to model the cluster for MSCD calculations. Normally, MSCD is applied to periodic surface structures. Even for some disordered surfaces, as in some adsorption systems, the substrate is still periodic. But for quasicrystals, both the surface and deeper layers are aperiodic. Hence the choice of suitable clusters for MSCD calculations has to be carefully considered and will be discussed in the next section.

The detailed theory and computational techniques underlying the MSCD code have been described in Ref. 15, so we only briefly review the method here.

In core-level photoemission, a photon illuminates an emitting atom and excites an electron from an atomic core level, ejecting the electron to a detector. The intensity of photoelectrons at the detector can be expressed in general as follows:

$$
I(\mathbf{K}, \theta, \phi) \propto\left|\phi_{0}+\sum_{j} \phi_{s j}\right|^{2} .
$$

Here $\mathbf{k}$ is the final electron wave vector, $\theta$ and $\phi$ are the polar and azimuthal angles of photoelectron emission, respectively, $\phi_{0}$ is the wave component representing travel along a path directly from the emitting atom to the detector without being scattered by another atom, and $\phi_{s j}$ is the scattered wave component representing travel via paths involving single or multiple scattering by one or more atoms, where $j$ represents the multiple scattering (MS) order. The multiple scattering expansion for spherically symmetric scatterers can be expressed in terms of diagonal plane-wave scattering $t$ matrices and matrix elements of the free-particle propagator expressed in an angular momentum and site basis. We use the RehrAlbers approximation: this approach expands the solution in terms of the "RA order," which can be adjusted to achieve convergence and limit the computational time. For most cases involving emission from an $s$ wave, it was found that the second RA order is adequate. ${ }^{15,20}$ More generally, for emission from an initial state $l_{i}$, the $\left(l_{i}+1\right)$ th RA order should be used for the first scattering event after emission; thereafter, the second RA order remains adequate. ${ }^{15}$

To better compare the angle-scanned curves in this work, the photoemission intensities of different polar and azimuthal angles are normalized to the $\chi$ function, ${ }^{15}$

$$
\chi=\left(I-I_{0}\right) / I_{0},
$$

where $I$ is the photoemission intensity at specific polar and azimuthal angles $(\theta$ and $\phi)$, and $I_{0}$ is the background subtracted from the intensity. For polar $\theta$ and azimuthal $\phi$ anglescanned curves, $I_{0}(\theta)$ and $I_{0}(\phi)$ are obtained by using a spline fitting method and a linear fitting method, respectively. ${ }^{15}$

The misfit between theory and experiment is quantified with a reliability factor ( $R$ factor), defined as

$$
R=\frac{\sum_{i}\left(\chi_{c i}-\chi_{e i}\right)^{2}}{\sum\left(\chi_{c i}^{2}+\chi_{e i}^{2}\right)},
$$

where $\chi_{c i}$ and $\chi_{e i}$ are calculated and experimental $\chi$ curves, respectively. We also use six other $R$ factors ${ }^{21}$ to confirm that the results do not depend on the particular form of $R$ factor chosen.

In view of the approximations that we must make to describe this infinitely complex surface, it should be borne in mind that the comparison between theory and experiment (as in Fig. 2, for example) must focus much more on the orientation of diffraction features (emission angles) than on their relative amplitudes. It should also be understood that diffraction features are frequently composed of multiple peaks, which in turn have relative amplitudes that are less reliable than their absolute orientations: so feature orientations are correspondingly more reliable than feature shapes.

\section{MODELING A QUASICRYSTAL SURFACE FOR MSCD CALCULATIONS}

The bulk structure of the AlPdMn quasicrystal used in this work is that determined by x-ray and neutron diffraction. ${ }^{2,3} \mathrm{~A}$ different, theoretical model is also available, ${ }^{22}$ but at our level of approximations we cannot distinguish between the two models. We take the bulk structure from a cube of AlPdMn with $100 \AA$ edges, centered at an arbitrary point. The quasicrystal surface is formed by cutting the bulk quasicrystal sample. If one cuts the sample at different positions along a fivefold axis, then one can achieve different surfaces consisting of various terminations of the bulk structure, as shown in Fig. 3. It can be seen that the composition, density, and geometric arrangement of atoms in the surface layers can be quite different for different terminations. This in fact is the central problem in all investigations of quasicrystal surfaces, since in a plane perpendicular to a quasicrystalline axis the concept of a lattice plane does not exist-in principle there are infinitely many atomic arrangements possible, although many of these will bear a close similarity to one another. Figure 4 shows the composition of several consecutive atomic planes and their interlayer spacings perpendicular to a fivefold axis based on the above bulk model.

As suggested by Figs. 3 and 4, no two atomic planes are identical. However, a subset of terminations exists which are all similar: this subset fits the experimental data best, as will be shown in the following sections. These terminations all consist of a bulklike Al-rich outermost layer, followed closely (about $0.48 \AA$ deeper into the bulk) by a mixed Al/Pd or $\mathrm{Al} / \mathrm{Pd} / \mathrm{Mn}$ layer. This "bilayer" is followed by more distant layers, and then by closer and denser layers. 


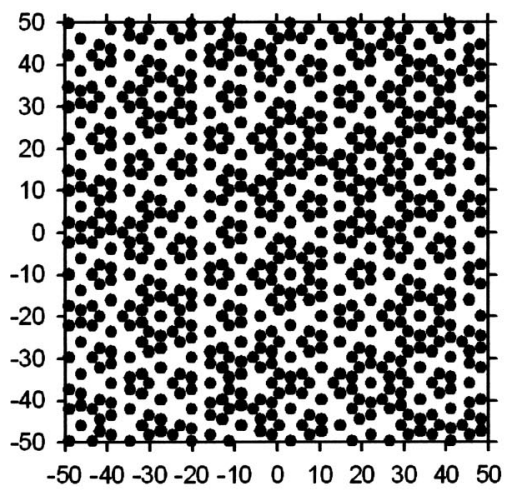

(a)

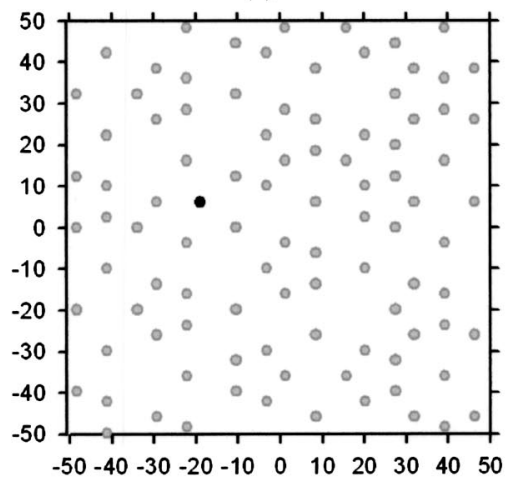

(c)

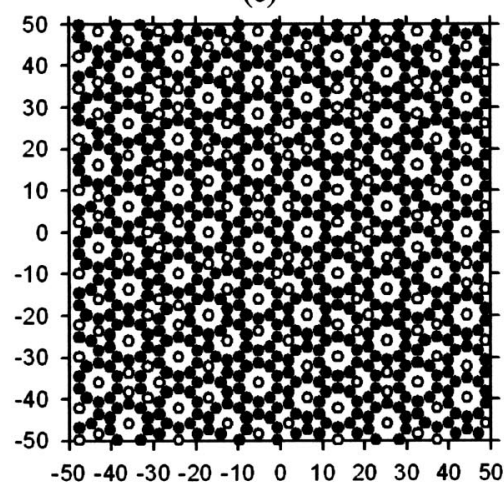

(e)

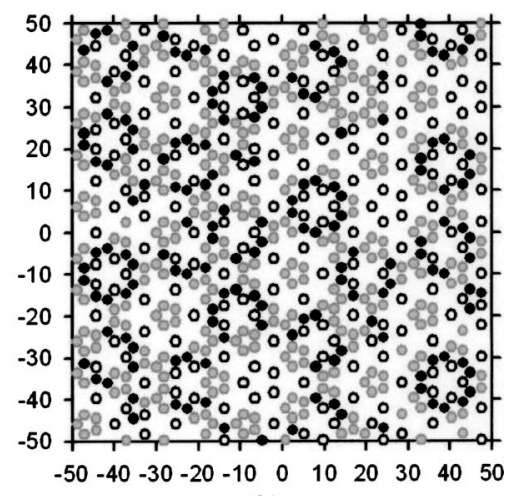

(b)

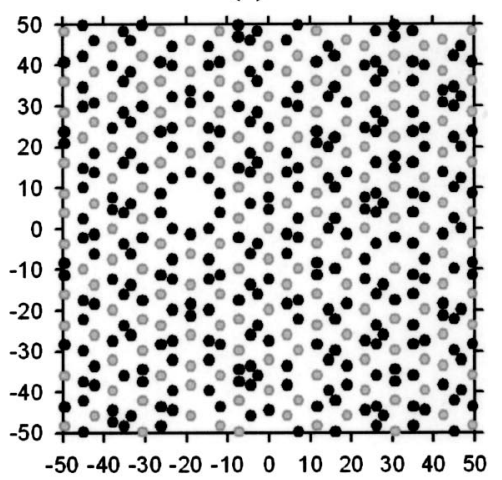

(d)

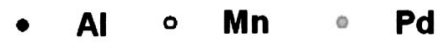

FIG. 3. Composition and atomic positions in a sequential set of typical planes which are perpendicular to the fivefold axis (axes are in angstroms). (a) is an Al-rich dense plane, followed by (b) another dense plane with mixed Al/Pd/Mn composition, then (c) a dilute layer mainly consisting of $\mathrm{Pd}$ atoms, and (d) a dense plane with mixed $\mathrm{Al} / \mathrm{Pd}$, and (e) a dense plane with mixed $\mathrm{Al} / \mathrm{Mn}$. The bulk interlayer spacings between successive planes (a)-(e) are 0.48, 0.78, 0.78, and $0.48 \AA$, respectively.
In MSCD calculations, the surface studied is represented by a half-ellipsoidal cluster of atoms whose shape takes into account the finite escape depth of photoelectrons due to inelastic scattering. The cluster dimensions are chosen to give convergence of the calculated results, to the extent that the computational cost remains acceptable.

The MSCD program relies on generating atomic positions through periodic lattice vectors, as appropriate for periodic crystals. For nonperiodic structures like quasicrystals, this approach can be extended by choosing a periodically repeated supercell which is larger than the cluster itself.

We next discuss the choice of cluster dimensions, as well as other parameters that enter the calculation. Tests were performed assuming Al $2 p$ emission. Representative calculated XPD patterns for the (unrelaxed) AlPdMn quasicrystal are shown in Fig. 5 as a function of cluster depth (from 2 to 12 layers). We choose a cluster radius of $12 \AA$, which is large enough for convergence, as shown in the following section. From Fig. 5, it can be seen that, when the number of layers $n$ reaches 4 , the simulated XPD pattern starts to show the five main spots of the experimental pattern. For $n>6$, the central spot appears, and the simulated patterns converge between $n=8$ and 12. In the later calculations, a cluster depth of about $5 \AA$ is used, which corresponds to about ten atomic layers. This depth may seem small compared to the inelastic attenuation length of about $12 \AA$, but it must be remembered that the total mean free path is smaller than this (due to elastic scattering). Also, the quasicrystalline structure involves "irregular" interlayer spacings and lateral positions, compared to more common metal surfaces, so that the emission from deeper layers tends to be forward scattered in many more directions by overlying layers, yielding a relatively diffuse contribution.

We also tested the lateral cluster dimension with radii ranging from $6 \AA$ (44 atoms within the cluster) to $15 \AA$ (299 atoms), and a depth of $5 \AA$. For the angular range considered here, the XPD patterns and $R$ factors converge when the cluster radius reaches $12 \AA$. Therefore, in the later simula- 


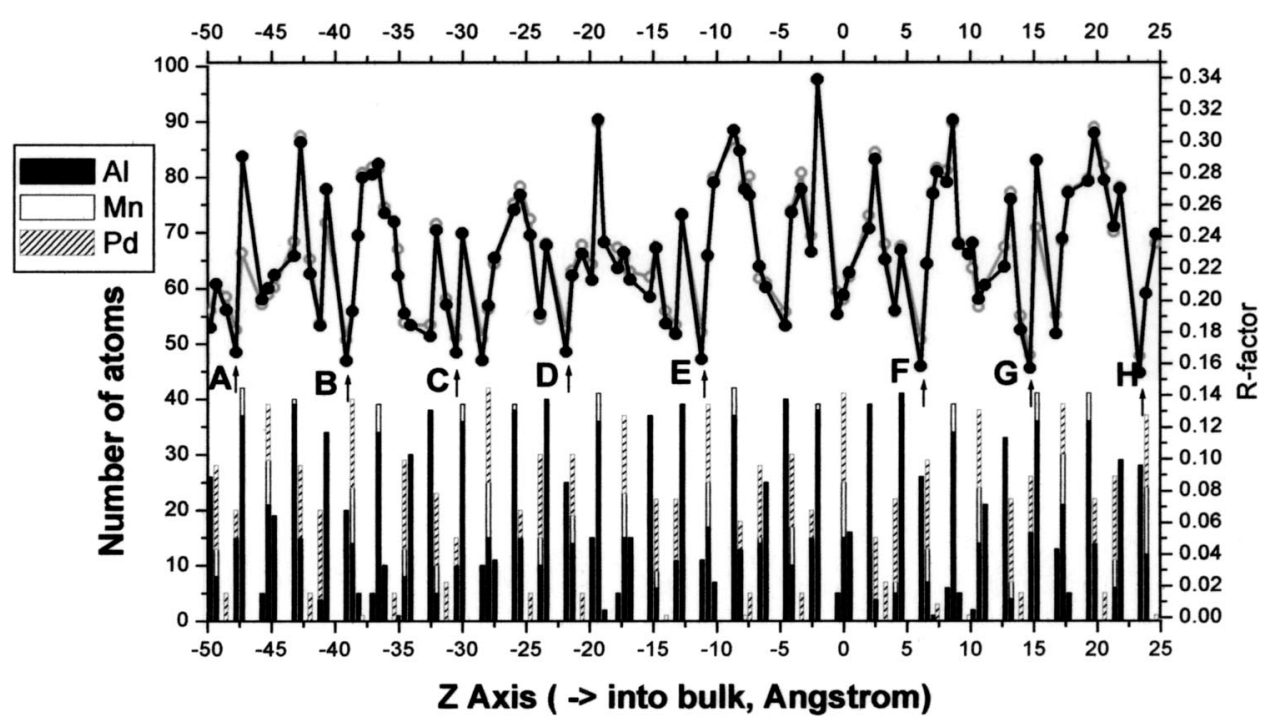

FIG. 4. Variation of $R$ factor with choice of different terminations for the AlPdMn quasicrystal: 100 consecutive terminations are included; a termination is defined by removing all layers to the left of a given layer (so positive $z$ points into the bulk, the origin being at an arbitrary position). The positions of bars represent individual bulklike atomic layers at their bulk positions along the fivefold axis, with different fill patterns defining their composition (see inset), and heights proportional to their two-dimensional atomic density (left ordinate gives number of atoms per $100 \times 100 \AA^{2}$ area). The lines connecting dots show $R$ factor values (right ordinate) for each termination. Unlike in Table I, these terminations are not optimized: the open dots assume bulklike interlayer spacings, while the filled dots relax the topmost and second interlayer spacings by -0.1 and $+0.1 \AA$, respectively. Arrows and letter labels identify those terminations that give the best $R$ factors: these terminations are interpreted as forming terraces.

tions, $12 \AA$ is chosen as the default radius of the clusters. Finally, we have optimized several nonstructural parameters that need to be taken to convergence: ${ }^{15}$ the multiple scattering order becomes $n_{\max }=4$, the RA order $|\mu|_{\max }=2$, the muffin-tin zero $V_{0}=4 \mathrm{eV}$, and the Debye temperature $\theta_{D}$ $=250 \mathrm{~K}$.

To start the structural analysis, we performed $\mathrm{Al} 2 p$ MSCD calculations for each of 100 possible terminations within a cube of surface area $100 \times 100 \AA^{2}$ and a depth of $100 \AA$, chosen arbitrarily to represent an average piece of bulk quasicrystal. Of 100 terminations studied, simulations of diffraction patterns for the three typical terminations shown in Figs. 3(a) -3 (c) are shown in Figs. 6(a)-6(c). Among these three, the surface terminated with a dilute Pd layer [Fig. 6(c)] gives the poorest agreement to experiment [repeated for $\mathrm{Al} 2 p$ from Fig. 2 in Fig. 6(d)]: the theoretical XPD shows ten strong spots in a ring, quite unlike the experimental pattern. In contrast, agreement is enhanced if the surface termination is one of the dense layers. From Fig. 6(a), it appears that a good match to features found in the experimental pattern is obtained by using as the outermost layer a dense $\mathrm{Al}(+\mathrm{Mn})$ layer; this notation means that the composition in the layer is mostly or totally Al, with perhaps some Mn [cf. Fig. 3(a)]. This is followed by a dense $\mathrm{Al} / \mathrm{Pd} / \mathrm{Mn}$ layer [with a mix of $\mathrm{Al}, \mathrm{Pd}$, and Mn; cf. Fig. 3(b)]. The strong differences between these three examples suggest that our MSCD calculations can indeed be used to determine which is the topmost layer of real quasicrystal surfaces, due to the fact that the calculated XPD pattern is very sensitive to the choice of termination.

Second, we have compared $R$ factors calculated via different definitions ${ }^{21}$ for a variety of terminations, to make sure that the best fit does not depend on how the $R$ factor is defined. For a cluster centered on a particular lateral site, different terminations in the $z$ direction (fivefold axis) are chosen as the topmost layer of the trial surface. We find that the trend of variation of $R$ factors with different terminations is the same for all the definitions of $R$ factors used for the MSCD calculations.

Third, we have to consider the lateral position of the emitter atoms, since all atoms of the same species, regardless of location, contribute to the total emission in our experiments. This corresponds to the choice of the location parallel to the surface of the ellipsoid that defines the selected cluster. Since the surface does not have a two-dimensional unit cell, there is an infinite variety of inequivalent emitter locations. As we cannot model all possible locations of the emitters, the strategy is to select only those clusters that occur predominantly on the surface. To that end, we tested four very different ellipsoidal clusters centered on various characteristic points of the surface (producing the required fivefold pattern symmetry by averaging over fivefold rotated orientations). We find that the variation of $R$ factors between these sites is significantly smaller than that between different terminations. This means that XPD is more sensitive to emitter location and thus surface structure in the perpendicular direction than in lateral directions for the fivefold quasicrystal surface. Consequently, only one lateral emitter location need be considered in each atomic layer below the surface.

\section{RESULTS}

The XPD of Al $2 p$ and Pd $3 d$ has been simulated using the MSCD code with the above optimized parameters. The 


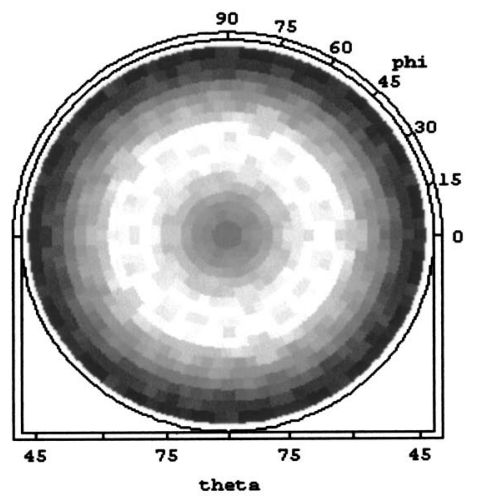

(2)

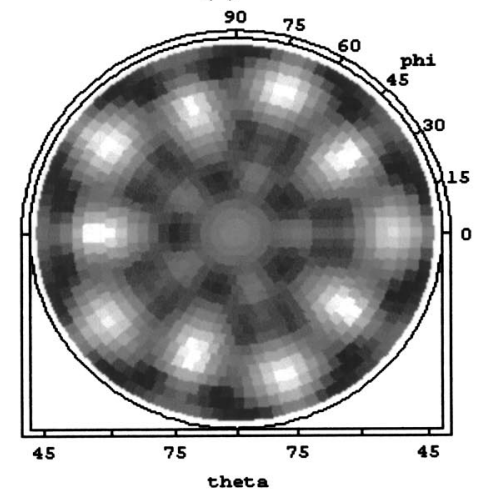

(6)

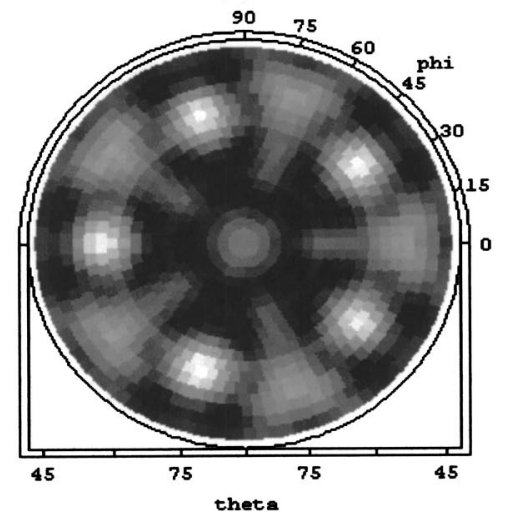

(10)

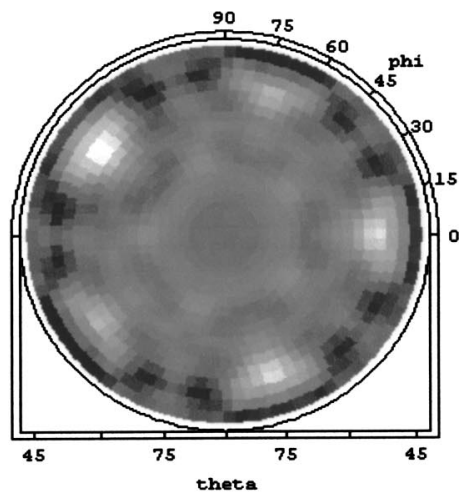

(4)

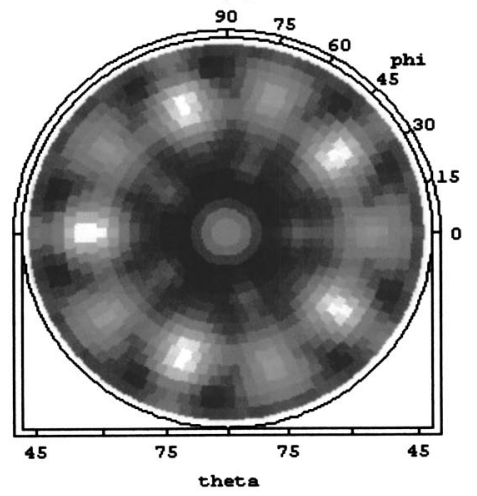

(8)

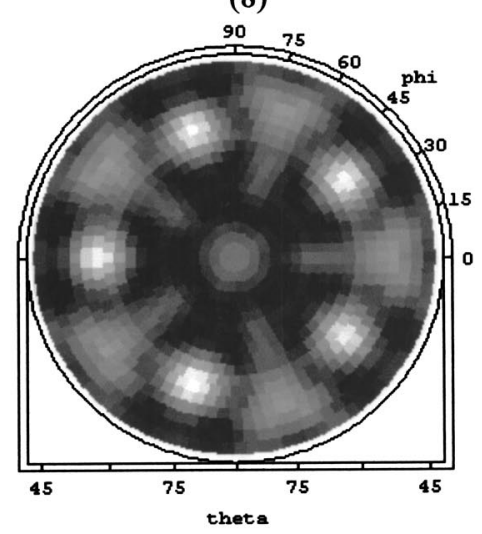

(12)
FIG. 5. Effect of depth of clusters on the XPD pattern for $\mathrm{Al} 2 p$ emission, shown for different numbers of layers from 2 to 12 . comparison between optimized simulation and the experimental data is shown in Fig. 2. The main features of the simulated patterns agree quite well with the experimental patterns. The simulated XPD patterns show clearly the fivefold symmetry, and the position and intensity of the main spots are close to those in the experimental data. We next discuss several structural aspects of this result.

\section{Favored terminations}

We have performed MSCD simulations for 100 clusters, each having a different termination, the results of which are summarized in Fig. 4. Several terminations with relatively good (small) $R$ factors are observed, as indicated with arrows and the labels $A-H$ in Fig. 4. It is found that those few terminations that best fit the experimental data have in common that they contain an Al-rich surface layer followed by a dense mixed $\mathrm{Al} / \mathrm{Pd} / \mathrm{Mn}$ layer: in each case, the outermost layer (marked by an arrow) is predominantly composed of $\mathrm{Al}$, while the second layer (to the right of the arrow) has a much more variable mixture of $\mathrm{Al}, \mathrm{Pd}$, and $\mathrm{Mn}$. These best terminations are not identical to each other. In a real quasicrystal surface, they may form terraces with many coexisting favorable terminations. It is therefore necessary to allow for mixtures of different terminations in the calculations, by averaging the diffraction patterns from the different terraces.

We have also explored a variety of random mixtures of different terminations, and considered averaging both over intensities and over $R$ factors from different terminations. The optimum structures mentioned below are found not to depend significantly on how the mix of terminations is handled.

Interestingly, Fig. 4 also shows a few other terminations that fit relatively well, if not as well as the first group dis- 


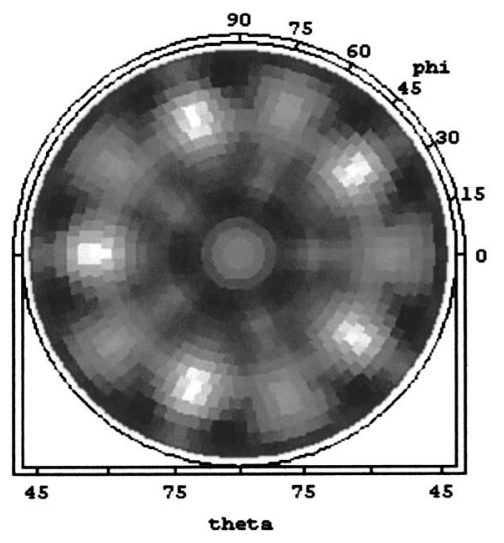

(a)

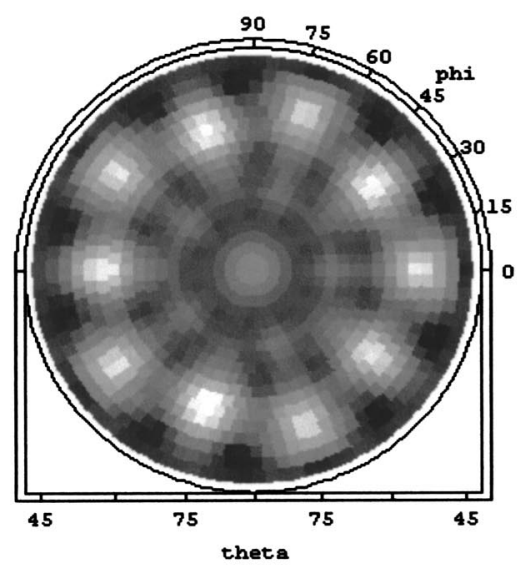

(c)

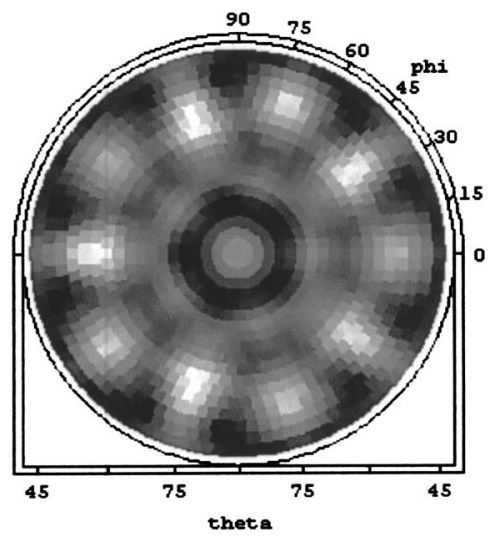

(b)

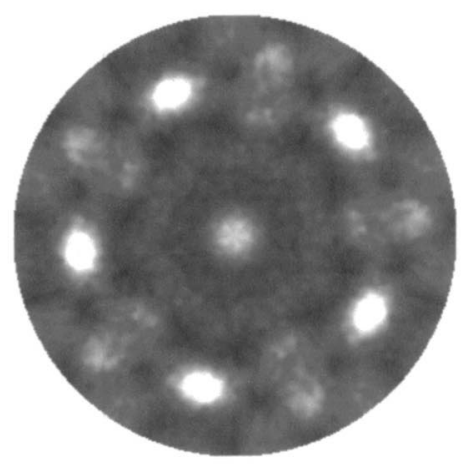

(d) cussed above: examples in Fig. 4 are the terminations with low $R$ factors occurring near $-46,-28,-11,-1$, and +17 $\AA$. These terminations consist of triplets of layers, with a mixed-composition higher-density central layer flanked by a pair of Al-rich but lower-density outer layers. They were also observed in the earlier LEED analysis, ${ }^{4}$ giving further support to the reliability of the results obtained with complementary techniques.

\section{Chemical composition}

Since XPD is an element-specific technique, it is useful to investigate the chemical composition from particular quasicrystal surface areas. To do this, we have used several typical best-fit clusters as examples and changed the chemical composition in the clusters by replacing $\mathrm{Pd}$ by $\mathrm{Al}$, or $\mathrm{Mn}$ by $\mathrm{Al}$, or some $\mathrm{Al}$ by either Pd or Mn. In these examples, we used Al $2 p$ as emitter to obtain XPD patterns and compared then with experimental data. We find that such clusters with modified compositions always give higher $R$ factors. The better (lower) $R$ factors are always obtained from clusters with a composition of $\mathrm{Al}(70 \pm 10 \%), \mathrm{Pd}(20 \pm 10 \%)$, and $\mathrm{Mn}$ $(10 \pm 10 \%)$, which is close to the bulk composition of the AlPdMn quasicrystal $(70.5: 21: 8.5)$. This indicates that the preparation technique used in the experiments actually does not produce excessive depletion of one atomic species against any of the others, in line with results from x-ray photoelectron spectroscopy.
FIG. 6. Comparison of theoretical simulated Al $2 p$ XPD for three different typical terminations with the experimental XPD patterns. (a) The topmost layer is a dense Al-rich layer (with or without Mn element in the same layer), followed by a dense mixed $\mathrm{Al} / \mathrm{Pd} / \mathrm{Mn}$ layer with interlayer spacing of $0.48 \AA$. (b) The topmost layer is a dense mixed $\mathrm{Al} / \mathrm{Pd} / \mathrm{Mn}$ layer followed by a dilute Pd layer with interlayer spacing of $0.48 \AA$. (c) The topmost layer is a dilute Pd layer, followed by a dense mixed $\mathrm{Al} / \mathrm{Pd}$ layer with interlayer spacing of $0.78 \AA$. (d) Experimental XPD of Al $2 p$ for comparison.

\section{Perpendicular relaxations}

To investigate the qualitative effect of variation of interlayer spacings on the $R$ factors, we first calculate the $R$ factor as a function of 100 individual possible terminations of an ideal (bulk) lattice. We then change the first and second interlayer spacings to $d_{12}-0.1 \AA$ and $d_{24}+0.1 \AA{ }^{23}$ according to previous LEED results, and then recalculate the $R$ factor. The results are illustrated in Fig. 4. It can be seen that for terminations with small $R$ factor, the values decrease slightly after modification of the interlayer spacing. This suggests that, for small- $R$-factor terminations, such a relaxation is favorable. Next, to determine more accurate relaxations of the interlayer spacings, a full optimization for these small- $R$ factor terminations is done. The optimized results of small$R$-factor terminations (as indicated with arrows in Fig. 4) are listed in Table I. The average $R$ factor is $\sim 0.16$, and the average best-fit interlayer spacing changes are $\Delta d_{12}$ $=-0.06 \pm 0.15 \AA$ (from $0.48 \AA$ in the bulk) and $\Delta d_{24}$ $=+0.16 \pm 0.15 \AA$ (from $1.56 \AA$ in the bulk).

The resulting spacing changes in this study by MSCD are in reasonable agreement with the previous LEED study, ${ }^{4}$ which determined the interlayer spacing changes of an AlPdMn quasicrystal to be $\Delta d_{12}=-0.06 \pm 0.04 \AA$ and $\Delta d_{24}$ $=+0.04 \pm 0.04 \AA{ }^{4,23}$ As noted above, that LEED study used a different preparation procedure, with a higher annealing temperature: brief annealing at $1050-1100 \mathrm{~K}$, followed by 
TABLE I. Optimum $R$ factors and best-fit changes in surface interlayer spacings (relative to the bulk) of an AlPdMn quasicrystal, by MSCD simulations. Results are shown for nine typical small- $R$-factor terminations with an Al-rich layer as surface topmost layer, and their average (terminations $A-H$ are shown in Fig. 4; termination $I$ would follow $H$ to its right). $\quad d_{24}$ is the interlayer spacing between the second dense layer and the next dense layer, which is the fourth layer. The third layer is a dilute Pd layer. Bulklike values of interlayer spacings: $d_{12}=0.48 \AA, d_{24}=1.56 \AA$. Unlike the $R$ factors shown in Fig. 4 , the values shown here were obtained by structural optimization. (Note: the LEED $R$ factor is included only for the sake of completeness; an XPD $R$ factor cannot be compared directly to a LEED $R$ factor.)

\begin{tabular}{lccccc}
\hline \hline Termination & Best-fit $R$ factor & $\begin{array}{c}\text { Best-fit } \Delta d_{12} \\
(\AA)\end{array}$ & $\begin{array}{c}\text { Best-fit } \Delta d_{12} \\
(\%)\end{array}$ & $\begin{array}{c}\text { Best-fit } \Delta d_{24} \\
(\AA)\end{array}$ & $\begin{array}{c}\text { Best-fit } \Delta d_{24} \\
(\%)\end{array}$ \\
\hline A & 0.1528 & 0.046 & +10 & 0.150 & +10 \\
B & 0.1543 & 0.067 & -14 & 0.052 & +3 \\
C & 0.1634 & -0.015 & -3 & 0.152 & +10 \\
D & 0.1588 & -0.204 & -43 & 0.198 & +13 \\
E & 0.1647 & 0.019 & +4 & 0.115 & +7 \\
F & 0.1549 & -0.170 & -35 & 0.255 & +16 \\
G & 0.1565 & -0.134 & -28 & 0.134 & +8 \\
H & 0.1522 & -0.132 & -28 & 0.250 & +16 \\
I & 0.1517 & 0.006 & +1 & 0.127 & +8 \\
Average & 0.1566 & $-0.06 \pm 0.15$ & $-12 \pm 30$ & $0.16 \pm 0.15$ & $+10 \pm 10$ \\
LEED (Ref. 4) & 0.31 & $-0.10 \pm 0.04$ & $-21 \pm 8$ & $0.04 \pm 0.15$ & $+3 \pm 10$ \\
\hline \hline
\end{tabular}

several hours of annealing at $870 \mathrm{~K}^{24}$ This results in flatter surfaces with larger terraces, as seen by STM and by sharper LEED patterns.

\section{Lateral relaxations}

We also consider lateral relaxations of the surface, i.e., atomic displacements parallel to the surface. We find a slightly better fit when the local structures are distorted with small lateral displacements of surface atoms. As an example, consider one typical best-fit termination [termination $D$; cf. Fig. 4 and Fig. 7(a)]: the magnitude of such a lateral displacement is about $5 \%$ of the nearest-neighbor distance within the layer. At the same time, the first interlayer spacing $\left(d_{12}\right)$ slightly increases by about $0.03 \AA$ to $0.31 \AA$ (still less

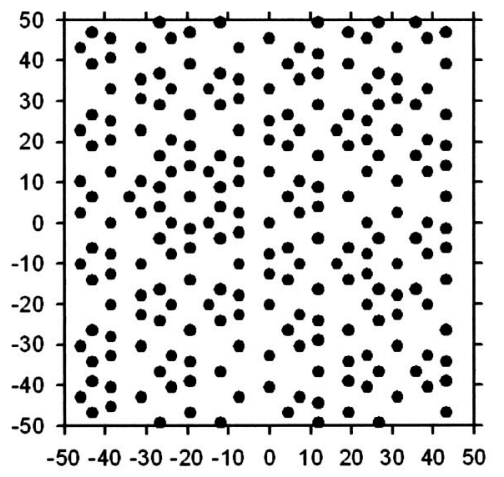

(a) Term. D

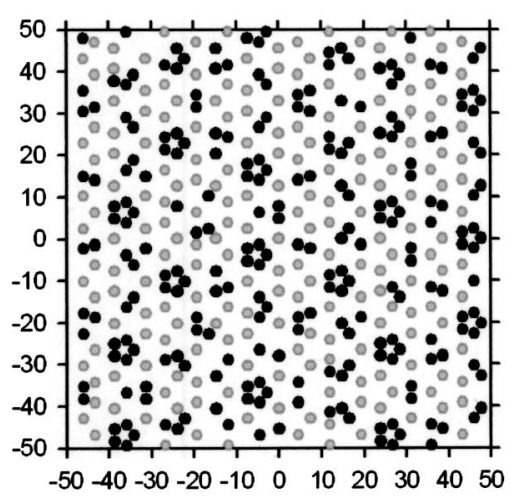

(c) Term. $\mathrm{H}$

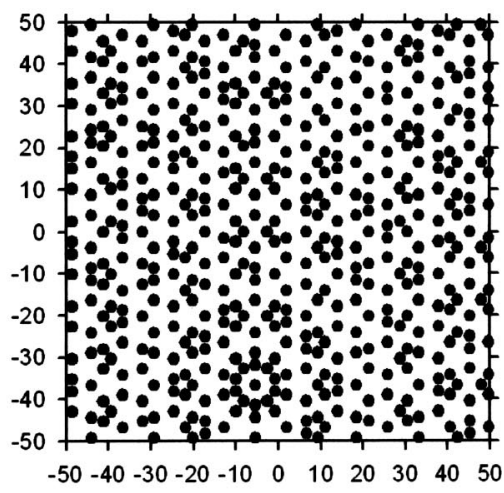

(b) Term. G

- Al ○ Mn $\quad$ Pd
FIG. 7. Three typical best-fit terminations, labeled as in Fig. 4 and drawn as in Fig. 3. 
than the bulk value $0.48 \AA$ ). The reduction in the $R$ factor is only 0.01 after such displacement: to verify the existence and magnitude of such relaxations would require additional data.

\section{DISCUSSION AND CONCLUSIONS}

In summary, we have compared experimental Al $2 p$ and Pd $3 d$ XPD patterns from a fivefold surface of icosahedral $\operatorname{AlPdMn}(000001)$ with a wide variety of XPD pattern simulations using multiple scattering calculations.

Simulations for 100 terminations of the bulk quasicrystal lattice produce several favorable terminations. These best terminations are not identical to each other, but show common features, namely, a dense Al-rich layer followed by another dense $\mathrm{Al} / \mathrm{Pd} / \mathrm{Mn}$ layer with average interlayer spacing of $0.42 \AA$ after optimization (compared to $0.48 \AA$ in the bulk). The quasicrystal surface also shows slight lateral displacements. Our results suggest that the real surface of the quasicrystal is likely formed by coexisting terminations, in the form of terraces separated by steps (as also $\operatorname{seen}^{5-7}$ by STM).

The present results can be compared with the (few) quantitative investigations of quasicrystal surface structure. The LEED study by Gierer et $\mathrm{ll}^{4}{ }^{4}$ has already been mentioned above. Naumovic et al..$^{25,26}$ have investigated the fivefold and twofold surfaces of icosahedral AlPdMn by full hemispherical x-ray photoelectron diffraction using the $\mathrm{Al} 2 s, \mathrm{Pd}$ $3 d_{5 / 2}$, and $\mathrm{Mn} 2 p_{3 / 2}$ photoemission lines. Comparing their patterns to single-scattering cluster simulations, ${ }^{27}$ they concluded that the environment of each element is very similar and has icosahedral symmetry. Their 51-atom clusters consisted of a pair of nested icosahedra around a central atom, and gave good qualitative agreement with $\mathrm{Al} 2 s$ diffraction, although no quantitative comparison in terms of $R$ factors was attempted (their good fit between theory and experiment despite a single-scattering model is related to the unique structure of quasicrystals: few atoms are lined up in straight chains as in a regular crystal, thereby reducing the multiple scattering that occurs chiefly along such chains of atoms at typical XPD energies). In view of the limited size of their model structure, information on different surface terminations and atom displacements in the immediate surface region, such as presented in Sec. IV above, was not obtained.

\section{ACKNOWLEDGMENTS}

We thank Dr. Y. Chen, Dr. R. Díez Muiño, Dr. B. S. Mun, and Dr. S.-H. Yang (LBNL, Berkeley) for helpful discussions. J.C.Z. thanks the Surface Science Lab of NUS and the Lee Foundation (Singapore) for financial support during his study leave at LBNL, Berkeley. The work was supported in part by the Director, Office of Science, Office of Basic Energy Sciences, Materials Sciences Division, of the U.S. Department of Energy under Contract No. DE-AC0376 SF00098.
${ }^{1}$ C. Janot, Quasicrystals: A Primer (Oxford University Press, Oxford, 1992).

${ }^{2}$ M. Boudard, M. de Boissieu, C. Janot, G. Heger, C. Beeli, H. U. Nissen, H. Vincent, R. Ibberson, M. Audier, and J. M. Dubois, J. Phys.: Condens. Matter 4, 10149 (1992).

${ }^{3}$ M. de Boissieu, P. Stephens, M. Boudard, C. Janot, D. L. Chapman, and M. Audier, J. Phys.: Condens. Matter 6, 10725 (1994).

${ }^{4}$ M. Gierer, M. A. Van Hove, A. I. Goldman, Z. Shen, S. L. Chang, C. J. Jenks, C. M. Zhang, and P. A. Thiel, Phys. Rev. Lett. 78, 467 (1997); M. Gierer, M. A. Van Hove, A. I. Goldman, Z. Shen, S. L. Chang, P. J. Pinhero, C. J. Jenks, J. W. Anderegg, C. M. Zhang, and P. A. Thiel, Phys. Rev. B 57, 7628 (1998).

${ }^{5}$ T. M. Schaub, D. E. Bürgler, H.-J. Güntherodt, and J. B. Suck, Z. Phys. B: Condens. Matter 96, 93 (1994).

${ }^{6}$ T. M. Schaub, D. E. Bürgler, H.-J. Güntherodt, J. B. Suck, and M. Audier, Appl. Phys. A: Mater. Sci. Process. 61, 491 (1995).

${ }^{7}$ P. Ebert, M. Feuerbacher, N. Tamura, M. Wollgarten, and K. Urban, Phys. Rev. Lett. 77, 3827 (1996).

${ }^{8}$ E. G. McRae, R. A. Malic, T. H. Lalonde, F. A. Thiel, H. S. Chen, and A. R. Kortan, Phys. Rev. Lett. 65, 883 (1990).

${ }^{9}$ Z. Shen, C. R. Stoldt, C. J. Jenks, T. A. Lograsso, and P. A. Thiel, Phys. Rev. B 60, 14688 (1999).

${ }^{10}$ G. Kasner, Z. Papadopolos, P. Kramer, and D. E. Bürgler, Phys. Rev. B 60, 3899 (1999).

${ }^{11}$ L. Barbier, D. Le Floc'h, Y. Calvayrac, and D. Gratias, Phys. Rev. Lett. 88, 085506 (2002).

${ }^{12}$ J. Ledieu, R. McGrath, R. D. Diehl, T. A. Lograsso, D. W.
Delaney, Z. Papadopolos, and G. Kasner, Surf. Sci. Lett. 492, L729 (2001).

${ }^{13}$ Z. Papadopolos, G. Kasner, J. Ledieu, E. J. Cox, N. V. Richardson, Q. Chen, R. D. Diehl, T. A. Lograsso, A. R. Ross, and R. McGrath, Phys. Rev. B 66, 184207 (2002).

${ }^{14}$ C. J. Jenks, A. R. Ross, T. A. Lograsso, J. A. Whaley, and R. Bastasz, Surf. Sci. 521, 34 (2002).

${ }^{15}$ Y. Chen and M. A. Van Hove, http://electron.lbl.gov/mscdpack/ mscdpack.html; see also: Y. Chen, F. J. García de Abajo, A. Chassé, R. X. Ynzunza, A. P. Kaduwela, M. A. Van Hove, and C. S. Fadley, Phys. Rev. B 58, 13121 (1998), and references therein.

${ }^{16}$ C. S. Fadley, Y. Chen, R. E. Couch, H. Daimon, R. Denecke, J. D. Denlinger, H. Galloway, Z. Hussain, A. P. Kaduwela, Y. J. Kim, P. M. Len, J. Liesegang, J. Menchero, J. Morais, J. Palomares, S. D. Ruebush, E. Rotenberg, M. B. Salmeron, R. Scalettar, W. Schattke, R. Singh, S. Thevuthasan, E. D. Tober, M. A. Van Hove, Z. Wang, and R. X. Ynzunza, Prog. Surf. Sci. 54, 341 (1997), and references therein.

${ }^{17}$ C. S. Fadley, M. A. Van Hove, Z. Hussain, A. P. Kaduwela, R. E. Couch, Y. J. Kim, P. M. Len, J. Palomares, S. Ryce, S. Ruebush, E. D. Tober, Z. Wang, R. X. Ynzunza, H. Daimon, H. Galloway, M. B. Salmeron, and W. Schattke, Surf. Rev. Lett. 4, 421 (1997), and references therein.

${ }^{18}$ D. Naumovic, P. Aebi, L. Schlapbach, C. Beeli, T. A. Lograsso, and D. W. Delaney, Phys. Rev. B 60, R16 330 (1999).

${ }^{19}$ R. S. Saiki, A. P. Kaduwela, M. Sagurton, J. Osterwalder, D. J. 
Friedman, C. S. Fadley, and C. R. Brundle, Surf. Sci. 282, 33 (1993).

${ }^{20}$ A. P. Kaduwela, D. J. Friedman, and C. S. Fadley, J. Electron Spectrosc. Relat. Phenom. 57, 223 (1991).

${ }^{21}$ M. A. Van Hove and R. J. Koestner, in Proceedings of the Conference on Determination of Surface Structure by LEED, edited by P. M. Marcus and F. Jona (Plenum, New York, 1984), p. 357.

${ }^{22}$ V. Elser, Philos. Mag. B 73, 641 (1996); A. Katz and D. Gratias, J. Non-Cryst. Solids 153-154, 187 (1993).

${ }^{23}$ We define $d_{24}$ to be the interlayer spacing between the second layer (dense) and the fourth layer (dense), equal to the original $d_{23}+d_{34}$ in the LEED study of Ref. 4 . Because the third layer is a dilute Pd layer, the contribution of its position displacement along the five-fold axis between the second and fourth layers to the total $R$ factor is relatively small, so its position is uncertain compared to $d_{24}$.

${ }^{24}$ C. J. Jenks, D. W. Delanay, T. E. Bloomer, S. L. Chang, T. A. Lograsso, Z. Shen, C. M. Zhang, and P. A. Thiel, Appl. Surf. Sci. 103, 485 (1996).

${ }^{25}$ D. Naumovic, P. Aebi, L. Schlapbach, and C. Beeli, in New Horizons in Quasicrystals: Research and Applications, edited by A. Goldman (World Scientific, Singapore, 1997), p. 86.

${ }^{26}$ D. Naumovic, P. Aebi, L. Schlapbach, C. Beeli, T. A. Lograsso, and D. W. Delaney, in Proceedings of the 6th International Conference on Quasicrystals, edited by T. Fujiwara and S. Takeuchi (World Scientific, Singapore, 1998), p. 749.

${ }^{27}$ See, for example, C. S. Fadley, in Synchrotron Radiation Research: Advances in Surface Science, edited by R. Z. Bachrach (Plenum, New York, 1989). 